\title{
Modularity and Symmetry in Computational Embryogeny
}

\author{
Or Yogev \\ California Institute of \\ Technology \\ 1200 East California Blvd. \\ Pasadena, CA 91125 U.S.A. \\ or@caltech.edu
}

\author{
Andrew A. Shapiro \\ Jet Propulsion Laboratory \\ California Institute of \\ Technology \\ 1200 East California Blvd. \\ Pasadena, CA 91125 U.S.A. \\ Andrew.A.Shapiro@jpl.nasa.gov
}

\author{
Erik K. Antonsson \\ California Institute of \\ Technology \\ 1200 East California Blvd. \\ Pasadena, CA 91125 U.S.A. \\ erik@design.caltech.edu
}

\begin{abstract}
Modularity and symmetry are two properties observed in almost every engineering and biological structure. The origin of these properties in nature is still unknown. Yet, as engineers we tend to generate designs which share these properties. In this paper we will report on the origin of these properties in three dimensional evolved structures (phenotypes). The phenotypes were evolved in an evolutionarydevelopmental model of biological structures. The phenotypes were grown under a high volatility stochastic environment. The phenotypes have evolved to function within the environment using the very basic requirements. Even though neither modularity nor symmetry have been directly imposed as part of the requirements, the phenotypes were able to generate these properties after only a few hundred generations. These results may suggest that modularity and symmetry are both very fundamental properties that develop during the early stages of evolution. This result may give insight to the origin of both modularity and symmetry in biological organisms.
\end{abstract}

\section{Categories and Subject Descriptors}

Categories and subject descriptors:I.2.11 Distributed Artificial Intelligence [Intelligent agents].

\section{General Terms}

Algorithms, Design

\section{Keywords}

Genetic Algorithm, Indirect Encoding, Stresses, Finite Element, Artificial Cell

\section{INTRODUCTION}

The origin of modularity in organisms has been observed in the early stages of evolution. Recent discovery of fossils indicates that modularity has been shared by ancient life

Copyright is held by the author/owner(s).

GECCO'08, July 12-16, 2008, Atlanta, Georgia, USA.

ACM 978-1-60558-130-9/08/07. forms 560 millions years ago [1]. One of the arguments corresponding to the rise of modularity, is a correlation with the robustness of the phenotype [2]. This argues for a possible relationship, between the ability of a phenotype to survive and the degree of modularity it poses. Modularity, in three dimensional forms, is very hard to quantify rigourously. Yet, as humans, we can easily determine whether an object is modular or not. In this paper we have created a three dimensional evolutionary and development model for structures [3]. The structures (phenotypes) were evolved to hold an external load in the form of wind. The wind has been exerted randomly on the phenotypes during their growth process. It can be shown that in the early stages of evolution the phenotypes look more like a blob of cells that tends to get organized with the process of evolution. In late stages of evolution, the phenotypes grow modules in the form of arms. These arms serve as supports for the phenotype and they tend to grow in a quasi-symmetrical way. The model presented here is an evolutionary development model (EvoDevo).

\section{ARTIFICIAL MODEL}

In the work reported here, an artificial embryogeny of structures has been created which is an extension of previous work done by the authors [3]. The two critical fundamental elements of this work are the selection of the artificial cell (the basic structural element) comprising each individual, and the artificial genes (the rules) which are evolved into the genetic information of each individual. The genetic information of an individual is shared by all of its cells. Each individual cell executes its rules until a mature structure is formed. Once maturity is reached, an evaluation scheme determines the fitness (performance) of the structure. Evolutionary operations (selection, crossover, and mutation) alter and refine genetic information in a population of individuals over multiple generations. The results are structures that meet the desired performance goals.

\subsection{Rules and Actions}

Mimicking nature, the basic structure of a gene is an ifconditional then-action rule. Every cell in our model can deform according to six different geometric operations: one for isotropic growth, two for anisotropic growth (B), three for shear $(\mathrm{S})$. In addition to the geometric operation actions, cell-type actions are defined. These actions are the three basic operations that occur in the developmental process of every biological structure, including; cell division, cell death, and cell differentiation. Cell division splits the cell into two 


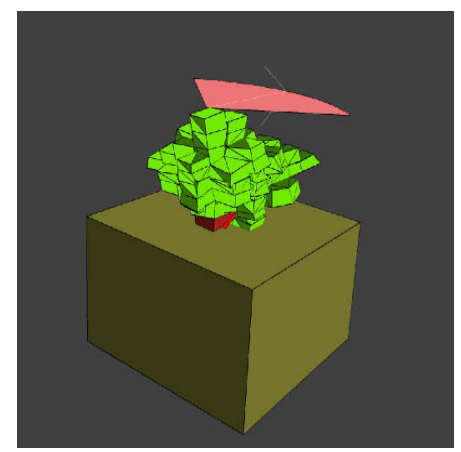

Figure 1: Phenotype at early stages of evolution - no degree of either symmetry nor modularity appear.

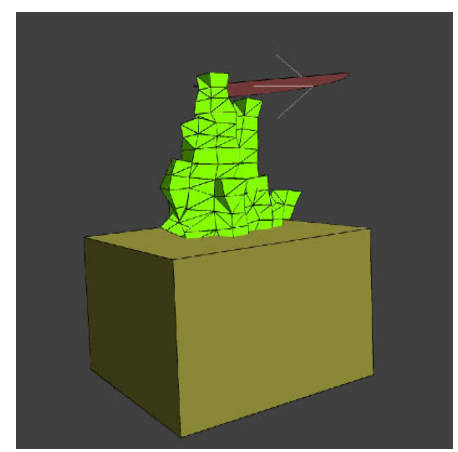

Figure 2: Phenotype at middle stages of evolution some degree of both modularity and symmetry begins to emerge.

equally sized cells, such that the total volume of the divided cells remains the same as that of the initial single cell. Cell death causes a cell to be removed from the model. Cell differentiation alters the material properties of a cell.

\subsection{Environment}

The environment in which the individuals are grown contains factors which every cell can sense and which may affect the way genes are expressed. The relationship between the information that cells receive from the environment and the development of the phenotype is not predetermined. Rather, conditionals are available to the evolutionary process that sense the concentration or gradient of each morphogen. In this way, the evolutionary process establishes the relationship between information and growth and development. In the artificial embryogeny presented here, two kinds of morphogens are present. The first represents a source that drives the growth of the phenotypes toward it. This morphogen is produced continuously at a predefined location and diffuses through space, impinging on the walls of each cell. The second morphogen represents the surface of the ground to which cells adhere when they intersect the surface.

\subsection{Results - Configuration and synthesis of structures}

The approach outlined above has been applied an experiment representative of an important problem in engineering and nature. The problem was to synthesize the configuration of a structure to support a highly varied load generated

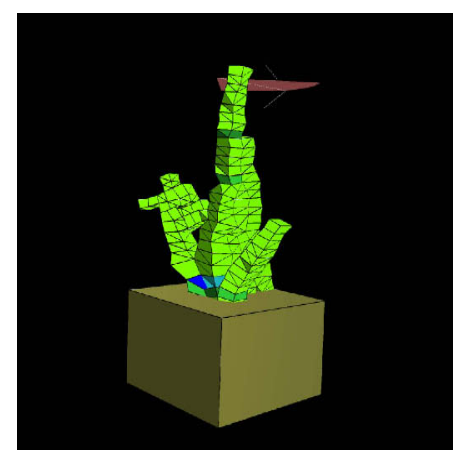

Figure 3: Phenotypes at the final stages of evolution - high degree of both modularity and symmetry begins to emerge. The phenotype contains modules which are symmetrical in two axes.

by a wind. In our model, the wind is not constant but rather changes randomly during the growth process. The goal of this experiment was to identify a relationship between the performance of the phenotype, subjected to a high volatility environment, and its degree of modularity and symmetry.

Figure 1 shows a phenotype in the early stage of evolution. The color of the cells represents their mechanical stress under the external loads (gravity, wind). The colors have been scaled from low to high, green - low stress, blue, gray - medium stress, and red - high stress. We noticed that the phenotype were mostly capable of supporting the load without been modular or symmetrical. Figure 2 shows a phenotype from at the middle stage of evolution. Unlike the early stages, this phenotype contains some degree of modularity and symmetry. Figure 3 shows a phenotype from the the late stage of evolution. We have notice that this phenotype has a high degree of symmetry and modularity.

\section{ACKNOWLEDGMENTS}

Our thanks to ACM SIGCHI for allowing us to modify templates they had developed. The research described in this paper was sponsored by the Jet Propulsion Laboratory, California Institute of Technology, under a contract with the National Aeronautics and Space Administration as part of the Ultra-Reliability Program.

\section{REFERENCES}

[1] G. M. Narbonn. Modular construction of early ediacaran complex life forms. Science, 305(5687):1141-1144, Dec. 2004.

[2] A. V. M. H. Peter Hammerstein, Edward H. Hagen and H. Herzel. Robustness: A key to evolutionary design. Biological Theory, pages 90-93, Dec. 2006.

[3] O. Yogev and E. K. Antonsson. Growth and development of continuous structures. GECCO200\%, pages 1064-1065, Dec. 2007. 